\title{
Performance Evaluation of QoS Parameters for Varied Network Overheads for Convergecast Routing in WSN
}

\author{
Lovepreet kaur $^{\mathrm{a}}$, Jyoteesh Malhotra ${ }^{\mathrm{b}}$ \\ ${ }^{\mathrm{a}}$ CSE Dept GNDU RC JAL, Batala, India \\ ${ }^{\mathrm{b}}$ CSE Dept GNDU RC JAL, Jalandhar, India
}

\begin{abstract}
Convergecast communication is used in many sensor applications. The flow of data in convergecast routing is from many to one i.e. many sensory nodes sense the data and send through parent child relation to the root node or sink node. Convergecast routing is always followed by broadcasting. In broadcast communication is from one to many and in convergecast it is from many to one. As sensor nodes have limited battery capacity main focus is to save energy. As compare to wired network wireless network is prone to attacks and it is less reliable than the wired network. This paper investigates the affect of header length of network layer on the BER, SNIR, backoff duration, SNR and latency of convergecast routing with the help of omnet++ simulator.
\end{abstract}

Index Terms: Wireless Sensor Network, Convergecast routing, header length, Omnet++ Simulator, Back off and BER.

(C) 2015 Published by MECS Publisher. Selection and/or peer review under responsibility of the Research Association of Modern Education and Computer Science

\section{Introduction}

Convergecasting in wireless sensor network is the process in which data is collected from a set of sensory nodes by the central node or sink node. It is proved that multihop communication perform much better than the single hop communication. In case of multihop communication energy is utilized i.e. for sending data to the sink node or base station less energy is consumed where as in case of single hop communication pattern each node sense the data and then directly send that data to the base station or sink node without the help of intermediate nodes, so more energy is depleted in single hop communication. Convergecast is multi hop communication technique used with broadcasting. Network is of tree like structure and there is a cantered root node after the set up of network each sensory node senses the data and sends to its neighbor node which is closer to the root/ sink node. Node which senses the data and transmits that data is known as a child node in the network and that data is forwarded to the node which is closer to the sink node it means the node which

* Corresponding author.

E-mail address: lovebatala@gmail.com 
receives that packet of data that node is known as a parent node. Root node in the network requests other nodes for some type of data by sending broadcast message. Child node then send its data to the parent node and parent node add its own sensed data with the child node data and send to their parent node, this process continue till the data reached to the root node or sink node[13].

Convergecast routing differs from broadcasting, in broadcasting data flows from single node to multiple nodes as one to many(as shown in figure 1 a) but as compared to convergecast in which data flows from many to one(as shown in figure1 $\mathrm{b}$ ).

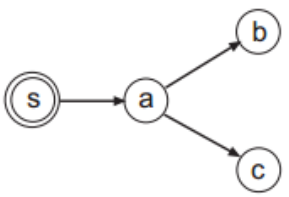

(a)

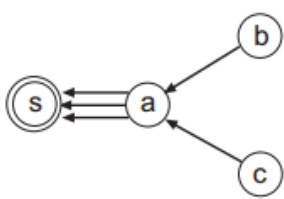

(b)

Fig.1. a) Broadcasting. b) Convergecasting.

Convergecast works on different network type but usually used with wise route network type. Wise route is a routing algorithm used in convergecast routing and in wireless sensor networks, this algorithm is a loop-free used in the routing tree construction from sink node to all sensory nodes, at first the center node (sink node) broadcast a message to the entire network for route building process. When each and every node got that broadcasted message they will resend that message and consider sender as the root node in the network. This process is basically used to join all the sensor nodes in the network so that they can communicate with each other i.e. part of synchronization. [10].In this sink node first send route packet to the network and when sensor node receives this route flood packet from sink node it check the RSSI (Received Signal Strength Indication) value, if it is higher then the previously stored threshold value the node select sending node as its parent node and if duplicate route packet with low RSSI value is received then sensor node simply discards that route flood message. Considering the merits as reported in the literature, the work done in this paper is mainly based on convergecast routing. In doing so the affect of network layer header length has been evaluated through the simulations. Rest of paper has been organized as follows:

In section 2 related works is discussed, in section 3 problem definition is defined, next section 4 gives brief simulation methodology. Performance results have been given in the section 5 before the paper is finally concluded in section 6 .

\section{Related work}

This section discusses the state of the art research being done in this field.

Ozlem Durmaz et al [1] analyzed two techniques like aggregated convergecast (where data is aggregated at each node) and raw data convergecast to collect the summarized information as a maximum sensors reading. Analyze the impact of different parameters like by using multiple frequencies; test the impact on packet loss and interference. This paper is about convergecast routing in wireless sensor network and by using TDMA (different time slots for each node to send data to the sink node) avoid number of collisions and avoid retransmission under heavy data .Various TDMA based scheduling algorithms are used in convergecast routing like minimize schedule length for faster data rate by reusing time slots again and again i.e. to decrease the number of slots per frame.

John Augustine Qi Han Philip Loden Sachin Lodha Sasanka Roy [15] This paper analysis how to choose shortest path to convergecast the data to sink i.e. many to one. This research test how the energy is saved by reducing the number of hops in convergecast to send the data to the sink node. By using shortest path finding algos path is selected to send data to sink node. 
Mrs.Aditi.P. Khadilkar et al[2] this research paper use the omnet++ and mixim framework to analyze the mobility based sensor networks and consider the MAC protocol to analyze various parameters like collision, loss of packets etc. and concluded with the help of omnet++ simulator that average energy consumption increases as the mobility of nodes increases because node are not stationary.

Andras Varga et al [3] omnet++ working environment is explained, the design of omnet++, history or background of simulator, understanding the architecture of omnet++, different types of files, how to use those files?, how the result stored in the result files?, how to change and compare different parameters in any network concluded with the overview of omnet++ simulator environment.

Nikolaos A. Pantazis et al [4] energy efficient routing protocols are classified into four main schemes: Network Structure, Communication Model, Topology Based and Reliable Routing. It is an analytical survey on energy efficient routing protocols for WSNs is provided.

M.Thangaraj et al [5] Routing basically flooding technique is discussed with the help of OMNET++ and MIXIM framework. In this paper various types of modules under the simulator are define like NED (Network Description Language) and .ini file. Various Flooding parameters are explained with the help of simulator like routing table, deployment of nodes, residual energy in wireless sensor network under the simulation environment and concluded how we change the already existing protocols accordingly and analyze the performance with the help of different parameters and also concluded that availability of research in omnet++ simulator is high because less expertise developers for codding.

A. Gogic.et al [6] impacts of routing techniques on wireless sensor network using omnet++ simulator MAC layer protocols are discussed. Flooding, gossiping, convergecast are analyzed by the battery charge or discharge value of capacitor. Battery level decreases as the number of nodes are increases, Packet delay is estimated using omnet++ simulator. Delay for different algorithms is calculated for WSN. Lai et al[14] use the greedy graph coloring technique is used to assign time slots to the sender to overcome the frequency interference problem in convergecast routing and find the shortest path to send data to the sink node so that throughput should increased. [16] Defines the security in data aggregation technique, concealed data aggregation in convergecast routing in wsn improves the security with energy efficiency in aggregation.

\section{Problem formulation}

In wireless sensor network broadcasting is followed by convergecasting process. Only few researchers work on issues of convergecast routing like latency improvement to get data faster and for reliable communication to decrease the bit error rate. This paper will simulates the effect of header length of network layer in convergecast routing, at different header lengths performance of convergecast routing in terms of various QoS parameters differs. For the selection of optimum size of header length simulative environment is created with OMNET++ simulator.

\section{Simulation Methodology}

To understand the various issues in WSN networks simulation environment is necessary, for this system new simulator based on discrete event simulation known as Objective Modular Network Test bed in C++ version4.4.1 (OMNET++) has been used. Performance of various nodes and networks are checked with the help of this simulator on the basis of different parameters in different scenario .Omnet++ is an extensible, modular, component based $\mathrm{C}++$ simulation library and framework, primarily for building network simulations. It is $\mathrm{C}++$ programming based communication model with GUI support. Due to its flexible architecture its application areas are high like simulation is used in various communication networks. Omnet++ is eclipse based IDE. It has text editor, $\mathrm{C}++$ based simulation launcher which helps in running simulation. Results for different scenario are stored in result files and plotted with the help of analyzer tool.

The convergecast routing protocol is modeled with the help of simulator indexed with mixim framework. The experiment is included on 9 sensor nodes with playground size $600 \mathrm{~m} * 600 \mathrm{~m}$. 
In figure 2.Initial deployment of nodes is shown as in running IDE.

Omnet++ is hierarchical based simulation model used for wired or wireless communication network. Nodes communicate with each other with the help of messages. The system contains the Network Definition File (NED File) which describes the basic structure of module with all the gates and parameters used in the module construction. GNED is one of the text editor used to write the NED code. Availability of different types of messages defined in .msg file. Translation of different messages is done through C++ library in OMNET++. Omnet.ini file is the main file in the simulator. Whenever program starts it reads the configuration file known as Omnet.ini. This file controls how the executions of simulator run, what are the parameters to be set and assign their values etc. The NED file is added in this configuration to import the structure of the network [7, 8 , 9].

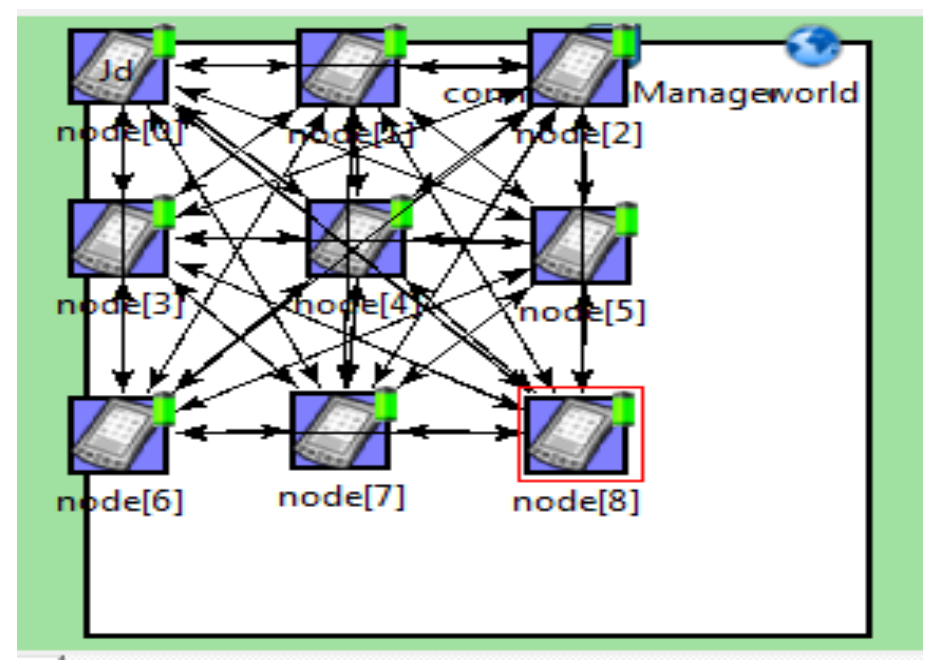

Fig.2. Deployment of Nodes.

Table 1. Simulation Parameters

\begin{tabular}{ll}
\hline Parameter & Value \\
\hline Number of sensor nodes & 9 \\
Carrier Frequency & $2.4 \mathrm{GHz}$ \\
Thermal Noise & $-100 \mathrm{dBm}$ \\
Header length & 16,24 and 32 bit \\
Mobility & Constant Speed \\
PMax (Sending Power) & $1.1 . \mathrm{mW}$ \\
Route flood interval & 600 seconds \\
Network type & Wise route \\
RSSI & $-50 \mathrm{dBm}$ \\
Simulation time & 10 min \\
\hline
\end{tabular}

Mixim is known as "mixed simulator" developed for wireless and mobile based simulations. This framework contains the detailed model for wireless networks and various communication protocols like MAC (Medium Access Control). Mixim has a powerful and feature rich tool box because of which the user can simulate and study the performance analysis of wireless networks. The specialty of Mixim is such that is tries to hide the 
complexity of such simulations and user gets a clean and easy user interface. Most of the simulators provide single frequency and single antenna systems, Mixim has rich library of protocols and modules also it has supporting infrastructure. Mixim can support simulation of networks consisting up to 1000 nodes [3].

\section{Result and Discussion}

Wise route is a routing algorithm used in convergecast routing and in wireless sensor networks, this algorithm is a loop-free used in the routing tree construction from sink node to all sensory nodes, at first the center node (sink node) broadcast a message to the entire network for route building process. When each and every node got that broadcasted message they will resend that message at consider sender as the root node in the network. This process is basically used to join all the sensor nodes in the network so that they can communicate with each other i.e. part of synchronization. [10].This is defined as route flood interval in simulator when the sink node sends the route packet to all the other nodes in the network. This simulation project analyses the different back off (retransmission of same data frames) on different route flood interval.

\subsection{Impact of header length on back off duration}

Result for this project in figure 3 shows that back off duration is very high when header length is 32 bit and retransmission i.e. back-offs are less at header length of 24 bit. So from the three variations of header length header length at 24 bit perform better in case of back off duration.

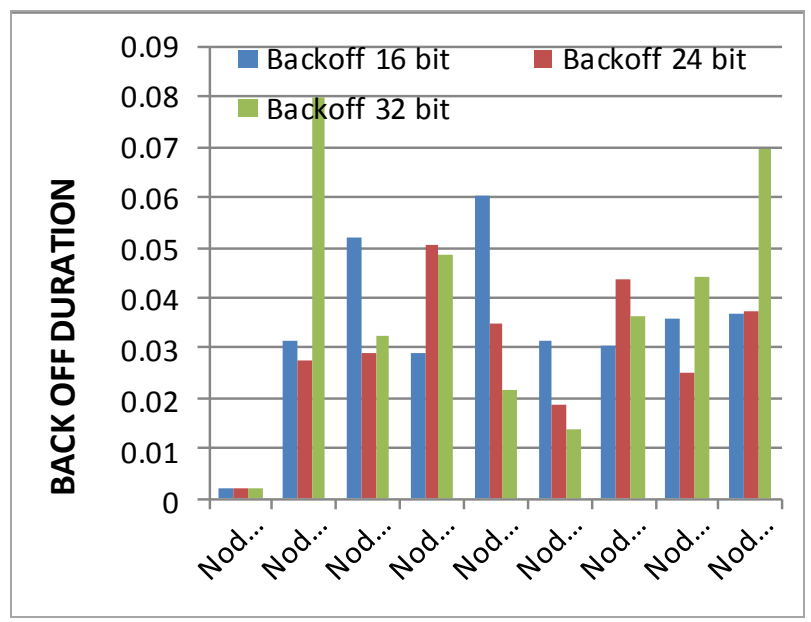

Fig.3. Back off duration at different RSSI

\subsection{Impact of header length of network layer on Signal to noise and Interference ratio}

Result for this project in figure 4 shows SNIR is high in case of 32 bit header length. SNIR increases with the increase in header length. At 16 bit header length signal strength is vey low and in 16 bit header length chances of errors are quite high. 


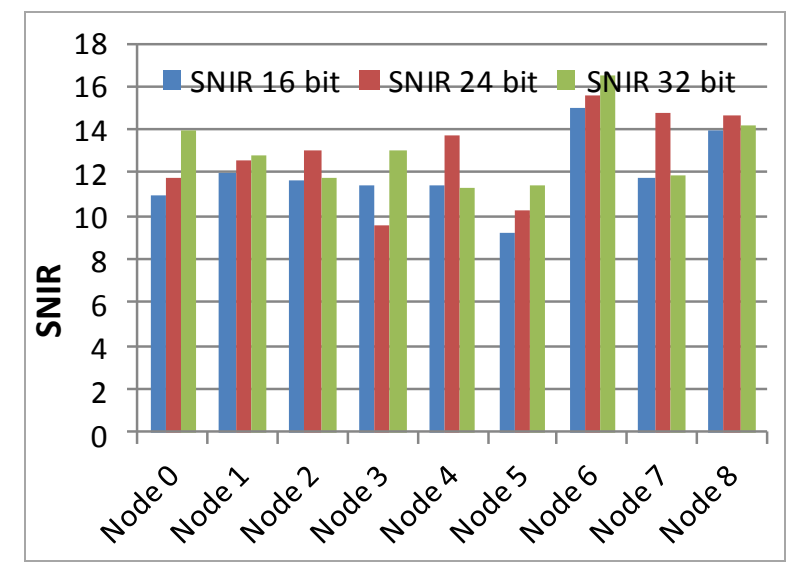

Fig.4. SNIR at Different Header Length of Network Layer.

\subsection{Impact of network layer header length on bit error rate}

From the simulative results it is shown BER (Bit Error Rate) is high in case of 16 bit header length. It means more bits get corrupted during communication in case of $16 \mathrm{HL}$. Noise and interference effect is high in case of 16 bit HL. Header length of 32 bit performs better as compare to 16 and 24 bit Header length.

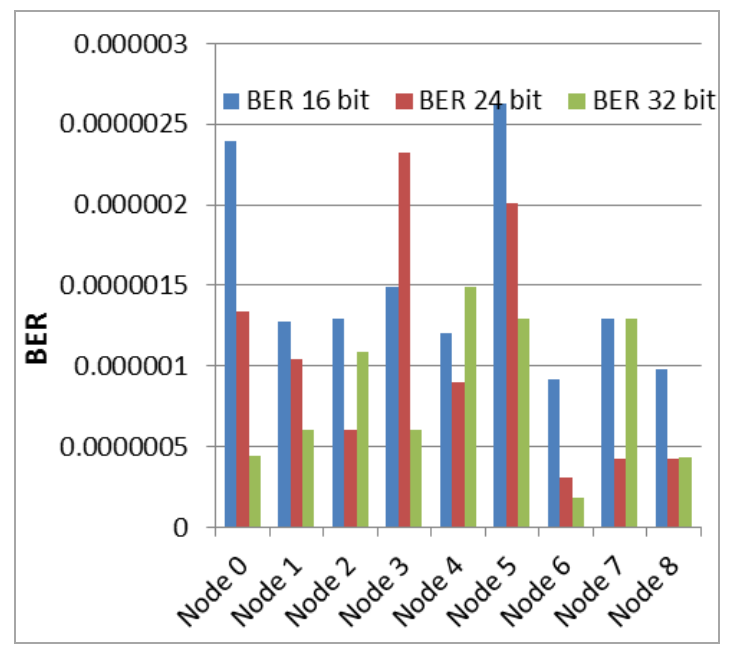

Fig.5. BER at 16, 24 and 32 Bit Header Length of Network Layer. Noise and Interference and Distortion Effect is Less in Case of 32 Bit Header Length.

\subsection{Impact of Network layer header length on SNR Signal to noise ratio Structure}

From the simulative results it is shown that in case Signal power to noise power ratio is high in 32 bit header length it shows that more useful information is available at 32 bit header length and in case of 16 bit header length SNR is very less as compare to 24 bit and 32 bit, which shows the presence of irrelevant and un-useful information i.e. presence of false information is more in case of 16 bit header length. 


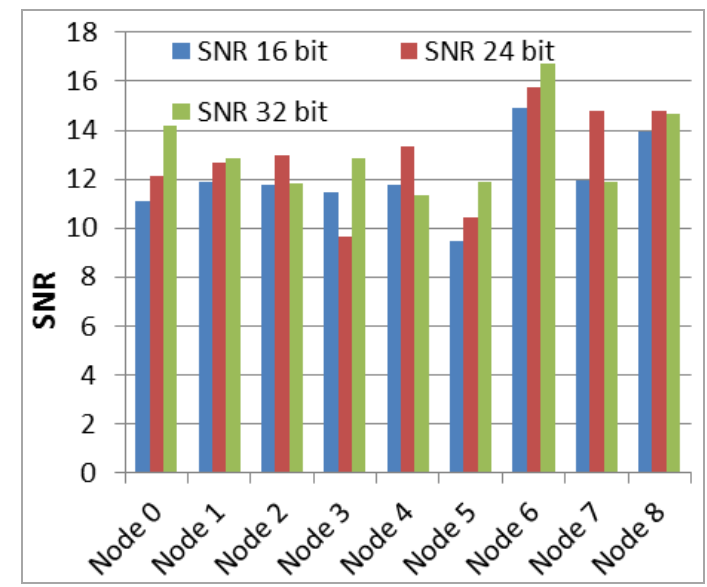

Fig.6. SNR at Different Header Length of Network Layer.

\subsection{Impact of network layer header length on Latency}

Latency is quite less in case of 32 bit header length which shows fast transmission or reception of data packet during communication process in convergecast routing as shown in fig. 7. Latency of network is the total time taken by the node to send the sensed data to the sink node or base station node.

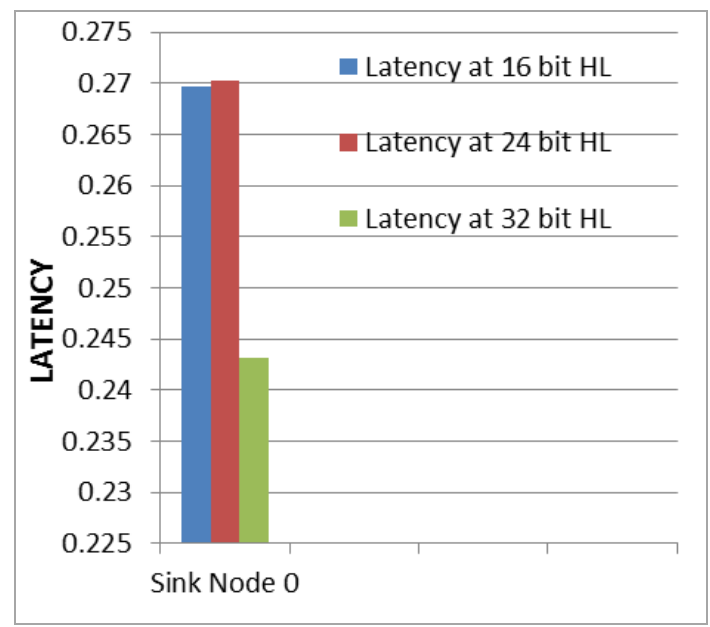

Fig.7. LATENCY at Different Header Length

\section{Conclusion}

Convergecast is a routing protocol mainly used to collect the sensed data from all the sensory nodes.so it is very important part for fast and reliable wireless communication in many sensor based applications. From this paper the simulative results concluded that with the increase of payload size upto 32 bit reliability of network increases as compare to 16 and 24 bit header length. Payload size directly affects on its performance i.e. better signal quality i.e. less distortion or interference is achieved at 32 bit header length and less delay exhibit at 32 
bit header length from the simulative analysis it is proved that in convergecast routing protocol optimum payload size is very important to save the energy and for better communication pattern but this protocol still needs researcher attention to solve various issues like security, energy depletion etc.

Table 2. Result Table

\begin{tabular}{|l|l|c|c|l|}
\hline \multicolumn{1}{|c|}{ Parameter } & 16 bit & 24 bit & 32 bit & \multicolumn{1}{c|}{ Rimulation Results } \\
\hline Back off & 0.310 & 0.269 & 0.348 & $\begin{array}{l}\text { In case of 32 bit header length back off duration is high which shows that fewer } \\
\text { collisions will occur if node senses the channel before transmission. }\end{array}$ \\
\hline SNIR & 107.5 & 115.96 & 116.9 & $\begin{array}{l}\text { Signal to noise and interference ratio increases with the increase in header length } \\
\text { i.e. up to 32 bit header length payload signal strength perform better. }\end{array}$ \\
\hline BER & .0000134 & .00000937 & .00000742 & $\begin{array}{l}\text { Bit error rate is high in case of low payload size and decreases with the increase in } \\
\text { payload size i.e. at 32 bit header length error rate reduce. }\end{array}$ \\
\hline SNR & 108.22 & 116.55 & 118.24 & $\begin{array}{l}\text { SNR increases with the increase in header length i.e. up to 32 bit header length } \\
\text { payload signal strength perform better. }\end{array}$ \\
\hline Latency & .269696 & .27024 & .243232 & $\begin{array}{l}\text { Latency increases in case of 16 and 24 bit because the more usage of bandwidth } \\
\text { and in case of 24 bit delay is less due to less traffic in communication and hence } \\
\text { the delay is also less. }\end{array}$ \\
\hline
\end{tabular}

\section{Acknowledgements}

First of all I would like to thank Dr Jyoteesh Malhotra for his support and valuable suggestions during the whole research process. No one can perform better without proper guidance. Further I am thankful to all faculty members and staff of department of computer science and engineering. Last but not the least I would like to thank my family members for their constant motivation and support.

\section{References}

[1] O.D Incel, A.Ghosh, B. Krishnamachari, and K. Chintalapudi "Fast data collection in tree based wireless sensor network," Journal IEEE Transactions on mobile computing-TMC vol 11 no.1,pp 86-99, 2012.

[2] A.P. Khadilkar, N. G. Palan "MAC protocol for mobile sensor network- modelling using omnet++ mixim network simulator,". (IJCSIT) International Journal of Computer Science and Information Technologies, Vol. 2 (3), 2011, 1154-1159.

[3] A.Varga and R.Hornig OpenSim “An overview of omnet++ simulator,” Open Sim Ltd.Sz 1 köz 11, 1032 Budapest, Hungary. SIMUTools, March 03 - 07, 2008, Marseille, France.ISBN 978-963-9799-20-2.

[4] Nikolaos A. Pantazis, Stefanos A. Nikolidakis and DimitriosD. Vergados, "Energy-Efficient Routing Protocols in Wireless Sensor Networks: A Survey" Communications Surveys \& Tutorials, IEEE (Volume:15, Issue: 225 July 2012.

[5] M.Thangaraj, S. Anuradha and P.Steffi Grace "A study of energy model in wsn routing using omnet++," International Journal of Application or Innovation in Engineering \& Management (IJAIEM) volume 3, issue 6, june 2014.

[6] A. Gogic,A.Mahmutbe govic, A.Mujcic, M.Zajc, I.Hakkı Cavdar, and N.Suljanovic "Impact of routing techniques on wireless sensor network using omnetpp," Original scientific paper Elektrotehni ski vestnik 80(4): 153-158, 2013.

[7] https://github.com. 
[8] http://www.omnetpp.org/

[9] http://mixim.sourceforge.net.

[10] Valliappan Annamalai "convergecasting in WSN" thesis Arizona state university. in: Proceedings of IEEE Wireless Communications and Networking Conference, 2003, Louisiana, USA.

[11] B.Sumalatha,T.Satish Babu "Node And Link Based Channel Assignment In WSN For Fast Convergecast" International Journal of Computer Trends and Technology (IJCTT) - volume 4 Issue10 Oct 2013.

[12] A. Kopke, M. Swigulski,K.Wessel, D. Willkomm P.T. Klein Haneveld T.E.V. Parker O.W. Visser H.S. Lichte, and S. Valentin "Simulating wireless and mobile network in omnet++ mixim vision" In Proc. Intl. Workshop on OMNeT++ (co-located with SIMU Tools '08), Mar. 2008.

[13] Q. Huang , Y. Zhang. Radial coordination for convergecast in wireless sensor networks. In Proc. IEEE 1st workshop on Embedded Networked Sensors (EmNeTS-I), 2004.

[14] N.lai, C. king and C.lin "On maximizing the throughput in convergecast in wireless sensor network", International conference, advances in grid and pervasive computing, (GPC 08) pp.396-408, 2008.

[15] John Augustine Qi Han Philip Loden Sachin Lodha Sasanka Roy "Tight Analysis Of Shortest Path Convergecast In Wireless Sensor network" Tata Research Development and Design Centre.

[16] Josna Jose and Joyse Jose "Asymetric concealed data aggragation techniques in WSNs: A Survey" International Journal of Information Technology and Computer science MECS, April 2014, Vol 5, Pages 28-25.DOI: 10.5815/ijitcs.2014.05.04.

\section{Author(s) Profiles}

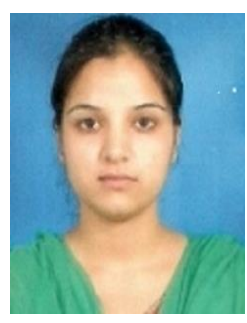

Lovepreet kaur was born in Batala city, state Punjab in INDIA during 9 Nov 1990. She completed Btech with Distinction from SSCET badhani, Pathankot and M.Tech from Guru Nanak Dev University (GNDU) RC Jalandhar during 2013-2015. Her research interests are in the broad area of QoS aware convergecast routing in wireless sensor network. She has published papers in scientific journals and also presented in international conferences.

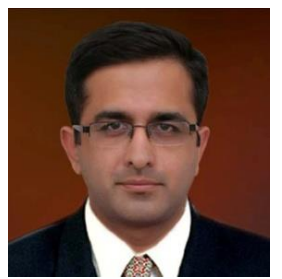

Dr. Jyoteesh Malhotra was born in Jalandhar, Punjab, INDIA. He completed B.Eng. with Distinction from P.R.M Institute of Technology \& Research, Amravati and M.Tech. with University Gold Medal from Guru Nanak Dev Engineering College, Ludhiana. He received $\mathrm{PhD}$ from Punjab Technical University in recognition to his contribution in the field of Wireless Communication \& Networks. From 1994 to 2007 he was employed with DAV CMC, New Delhi as Lecturer and Punjab University, Chandigarh as Assistant Professor. He joined Guru Nanak Dev University Regional Campus at Jalandhar in July 2007 where he is currently Associate Professor and Head of ECE \& CSE Departments.

His research interests are in the broad area of Pervasive Communication systems and Networks with emphasis on Statistical modelling of Fading Channels, Fading mitigation techniques, Optimization of High data rate Optical and wireless Communication Systems, Enhancement of QoS aware Wireless networks and Wireless Security. Dr. Malhotra has published and presented more than 100 technical papers in scientific journals and international conferences and authored 02 books. He is a life member of Indian Society for Technical Education (I.S.T.E.) and Editorial Board of many International Journals of repute. 